\title{
Lumped-Element Impedance-Transforming Uniplanar Transitions and Their Antenna Applications
}

\author{
Yo-Shen Lin, Member, IEEE, and Chun Hsiung Chen, Fellow, IEEE
}

\begin{abstract}
Novel impedance-transforming coplanar waveguide (CPW)-to-coplanar stripline transitions and CPW-to-slotline transitions are proposed, using planar lumped elements to realize the $L$-section impedance-matching network in the transition structures. Simple equivalent-circuit models based on closed-form expressions are also established, from which various lumped-element impedance-transforming transitions are investigated. Examples for using the proposed transitions in the design of antenna feeding structures are also demonstrated, and successful size reduction has been achieved.
\end{abstract}

Index Terms-Antenna feeding structure, impedancetransforming transition.

\section{INTRODUCTION}

C OPLANAR waveguide (CPW), coplanar stripline (CPS), and slotline (SL) are widely used as the building blocks for uniplanar monolithic microwave integrated circuits (MMICs) [1]. Many circuit components based on these uniplanar lines such as filters, couplers, mixers, and antenna feeding structures have also been proposed in recent years. To fully utilize the distinct features of CPWs, CPSs, and SLs, effective transitions or interconnections between them is of practical importance. Various CPW-to-CPS and CPW-to-SL transition structures were proposed and investigated [2]-[10]. Applications of these uniplanar transitions in balanced mixers, antenna feeding structures, frequency doublers, and CPW-to-waveguide transitions were also widely documented in the literature. Most of the previous studies on uniplanar transition design were focused on bandwidth improvement [2]-[5] or size reduction [4], [7]-[10]. In certain applications, the transition output is connected to other circuit components like mixer diodes [11] or antennas [12], [13] with different impedance values so that additional output matching or impedance transformation is required. However, previous studies on the impedance transformation of uniplanar transitions are rather limited.

Most of the conventional uniplanar transitions were designed with the same source and load impedances [2]-[10]. Simple impedance transformation was usually accomplished by adding a quarter-wavelength $(\lambda / 4)$ transformer in front of or after the transition [12], [13], therefore, the resulting bandwidth is

Manuscript received July 24, 2003; revised December 30, 2003. This work was supported by the Ministry of Education and by the National Science Council of Taiwan under Grant 89-E-FA06-2-4 and Grant NSC 92-2213-E-002-041.

The authors are with the Department of Electrical Engineering, Graduate Institute of Communication Engineering, National Taiwan University, Taipe 106, Taiwan, R.O.C. (e-mail: chchen@ew.ee.ntu.edu.tw).

Digital Object Identifier 10.1109/TMTT.2004.825670 narrow. The printed third- and fourth-order Marchand baluns in [3] may provide wide-band impedance transformation through the additional $\lambda / 4$ lines at one or both ends of the transition. The impedance-transforming Marchand balun [14], [15] may also achieve wide-band impedance transformation by adjusting the coupling coefficients of two $\lambda / 4$ coupled-line sections. However, these transitions/baluns occupy a large circuit area and are limited to the transformation of real impedance values, a consequence of employing $\lambda / 4$ lines. Thus, they may not be suitable in MMIC design, especially for low-frequency applications.

In [8], the planar lumped-element $L C$ circuits were used to replace the $\lambda / 4$ open or short stubs in conventional uniplanar transitions to reduce their sizes. In [9] and [10], the planar lumped elements were adopted to implement compact uniplanar transitions with filter characteristics. However, these lumped-element uniplanar transitions are limited to applications with the same source and load impedance values. In this paper, novel impedance-transforming uniplanar transitions are proposed by using planar lumped elements to realize the $L$-section matching network in the transition structure. The resulting transitions have very compact sizes and may provide impedance transformation to complex impedance values. For design purposes, simple equivalent-circuit models are also established. Applications of the proposed impedance-transforming transitions to the design of antenna feeding structures are also demonstrated.

\section{IMPEDANCE-TRANSFORMING CPW-TO-CPS TRANSITIONS}

\section{A. Type-I Structures}

Probably the simplest type of matching network is the $L$-section, which uses two reactive elements to match an arbitrary load impedance to a transmission line [16]. There are four possible configurations for this network, as shown in Fig. 1. If the normalized load impedance $z_{L}=Z_{L} / Z_{0}$ is inside the $1+j x$ circle on the Smith chart, then the circuits of Fig. 1(a) and (b) should be used. On the other hand, if the normalized load impedance is outside the $1+j x$ circle on the Smith chart, the circuits of Fig. 1(c) and (d) should be used.

Shown in Fig. 2(a) is the proposed impedance-transforming CPW-to-CPS transition to realize the $L$-section network in Fig. 1(a). Specifically, the series inductor $L_{s}$ is realized by a shorter metal strip and is series connected to the CPW center conductor. The shunt capacitor $C_{p}$ to ground is accomplished by the interdigital structure and is shunt connected to the left of 


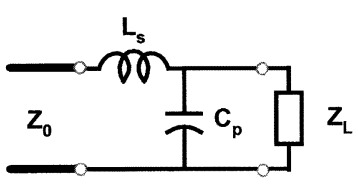

(a)

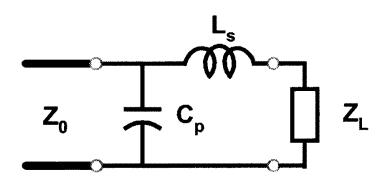

(c)

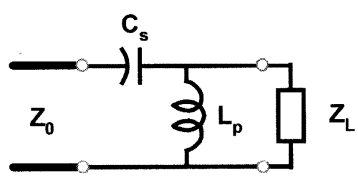

(b)

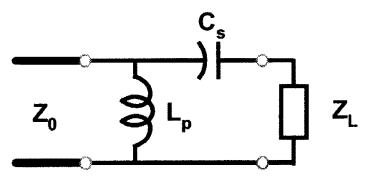

(d)
Fig. 1. $L$-section matching networks. (a) and (b) Networks for $z_{L}\left(=Z_{L} / Z_{0}\right)$ inside the $1+j x$ circle. (c) and (d) Networks for $z_{L}$ outside the $1+j x$ circle.

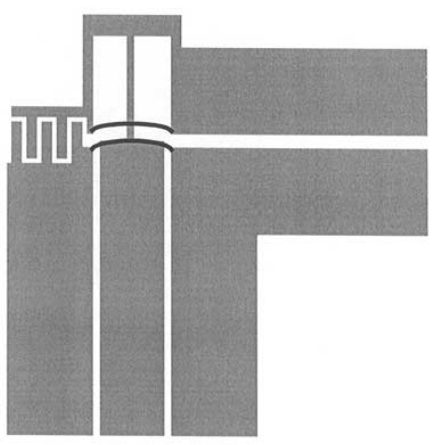

(a)

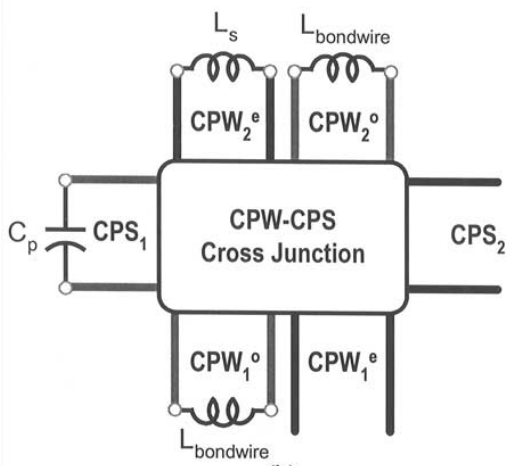

(b)

Fig. 2. Type-I impedance-transforming CPW-to-CPS transition that realizes the $L$-section network in Fig. 1(a). (a) Layout. (b) Equivalent-circuit model.

the CPW-CPS cross-junction. This arrangement may reduce the transition size since the series element along the transmission line usually occupies a larger area. For suppressing the odd CPW mode excited at the CPW-CPS cross-junction, bondwires at suitable positions are included. The resulted transition structure is then an L-section matching network with one port in the CPW and the other port in the CPS. Let the load impedance connected to the CPS port of the transition be $Z_{L}$, this transition structure is capable of transforming $Z_{L}$ to $Z_{\mathrm{cpw}}$ if the normalized load impedance $z_{L}=Z_{L} / Z_{\text {cpw }}$ is inside the $1+j x$ circle on the Smith chart. Shown in Fig. 3(a) is another realization of the $L$-section in which the first reactive element is a capacitor, as in Fig. 1(b). One may choose between the transition structures for Figs. 2(a) or 3(a) depending on the realizable range of the lumped elements and the requirement on dc blocking of the matching network.

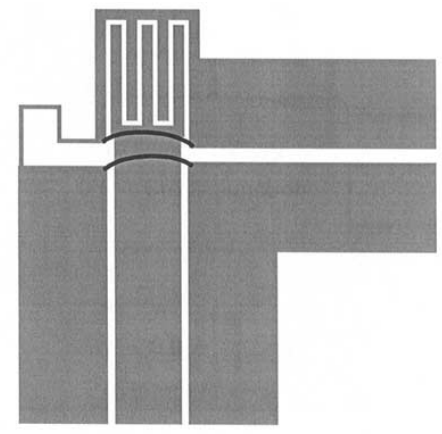

(a)

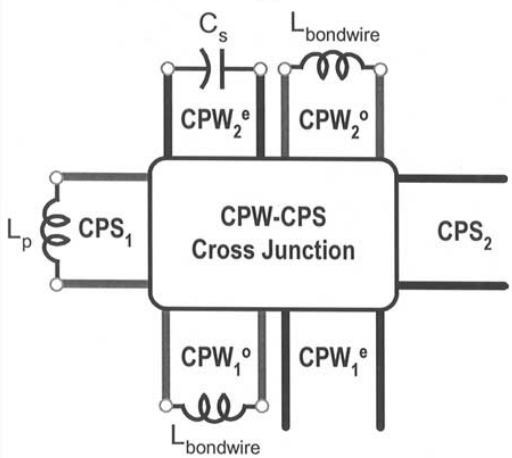

(b)

Fig. 3. Type-I impedance-transforming CPW-to-CPS transition that realizes the $L$-section network in Fig. 1(b). (a) Layout. (b) Equivalent-circuit model.

Characterization of the metal-strip inductance may be accomplished by the partial-element equivalent-circuit method [17] based on quasi-static assumptions. The inductance is obtained once the strip width, length, and thickness are specified. For calculating the capacitance of the interdigital structure, the conformal mapping technique under quasi-static assumptions [18] is adopted. The per-unit-length capacitance is first obtained and is then multiplied by the finger length to give the total capacitance. In order to take into account the mode conversion effect at the CPW-CPS cross-junction, a six-port circuit model [19], as shown in Fig. 4, is adopted. Specifically, each CPW line is represented by two transmission lines that support the even and odd CPW modes, respectively. Bondwires are modeled as inductors with the inductance values estimated as a function of their lengths and diameters. Thus, the incomplete suppression of the odd CPW mode at the CPW-CPS junction due to the finite bondwire inductance may then be suitably modeled. By adopting the six-port cross-junction model, the resulted equivalent-circuit models for the proposed impedance-transforming transitions [see Figs. 2(a) and 3(a)] may then be constructed as shown in Figs. 2(b) and 3(b). Here, the input CPW line and series reactance $L_{s}$ (or $C_{s}$ ) are connected to the even CPW mode ports $\mathrm{CPW}_{1}^{e}$ and $\mathrm{CPW}_{2}^{e}$ of the six-port junction model, respectively, while the bondwire inductances are used to terminate the odd CPW mode ports $\mathrm{CPW}_{1}^{o}$ and $\mathrm{CPW}_{2}^{o}$ at their corresponding positions. The shunt reactance $C_{p}$ (or $L_{p}$ ) and the output CPS line are connected to the CPS mode ports $\mathrm{CPS}_{1}$ and $\mathrm{CPS}_{2}$ on the left- and right-hand sides, respectively. These transition models [see Figs. 2(b) and 3(b)] are based on three assumptions. First, the CPW and CPS sections are modeled as transmission lines. Second, the discontinuity effect of the CPW-CPS 


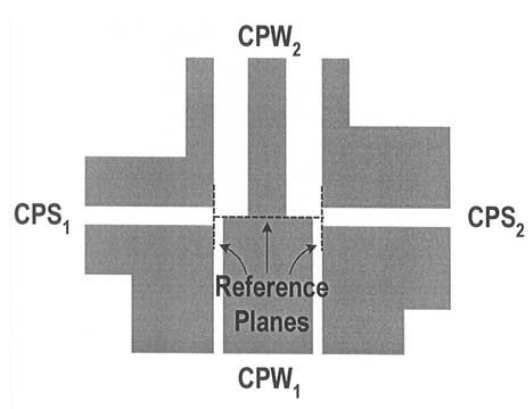

(a)

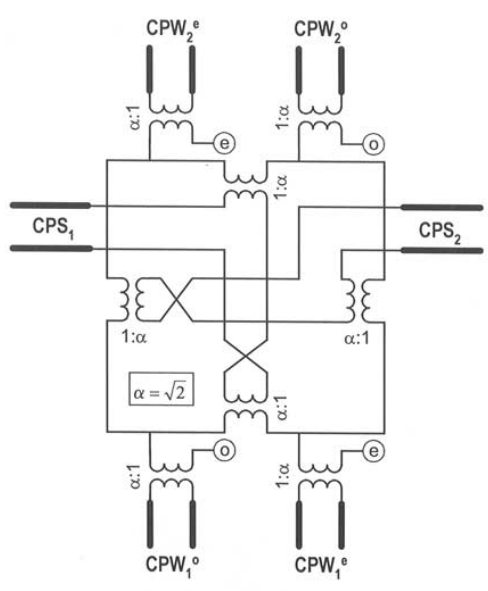

(b)

Fig. 4. CPW-CPS cross-junction. (a) Layout. (b) Six-port circuit model.

cross-junction is neglected. Third, the parasitic effects of the lumped elements ( $L$ and $C$ ) are neglected. Note that all the elements in the transition equivalent-circuit model are characterized by closed-form expressions with element values directly related to geometrical parameters. Therefore, the model is scalable and the simulation time may be drastically reduced.

The design of proposed impedance-transforming transition may be easily done through the basic knowledge on impedance transformation and the equivalent-circuit model. According to the given transition source and load impedances, the required $L$ and $C$ values are obtained through the Smith-chart calculations. The transition equivalent-circuit model may then be built in a circuit simulator, and simulation is performed to see whether the element values need to be fine tuned with the presence of the cross-junction model. After the element values are fixed, the geometrical parameters of the interdigital capacitor and metal strip inductor are obtained directly from the design formulas. The transition prototype may then be constructed.

A Type-I impedance-transforming transition for Fig. 2(a) is built on an FR4 substrate $\left(\varepsilon_{r}=4.3, \tan \delta=0.022\right.$, and thickness $h=1.6 \mathrm{~mm}$ ) in a back-to-back configuration. This transition is designed to transform the $50-\Omega$ CPW to $100-\Omega$ CPS with a center frequency of $2 \mathrm{GHz}$. The CPW line has a strip width of $3 \mathrm{~mm}$, slot width of $0.3 \mathrm{~mm}$, and finite ground-plane width of $4 \mathrm{~mm}$. The characteristic impedance of the CPW is $50 \Omega$ according to the closed-form formulas in [1]. The CPS line has a strip width of $4 \mathrm{~mm}$ and a slot width of $0.6 \mathrm{~mm}$ such that its characteristic impedance is $100 \Omega$ according to [1]. The CPS

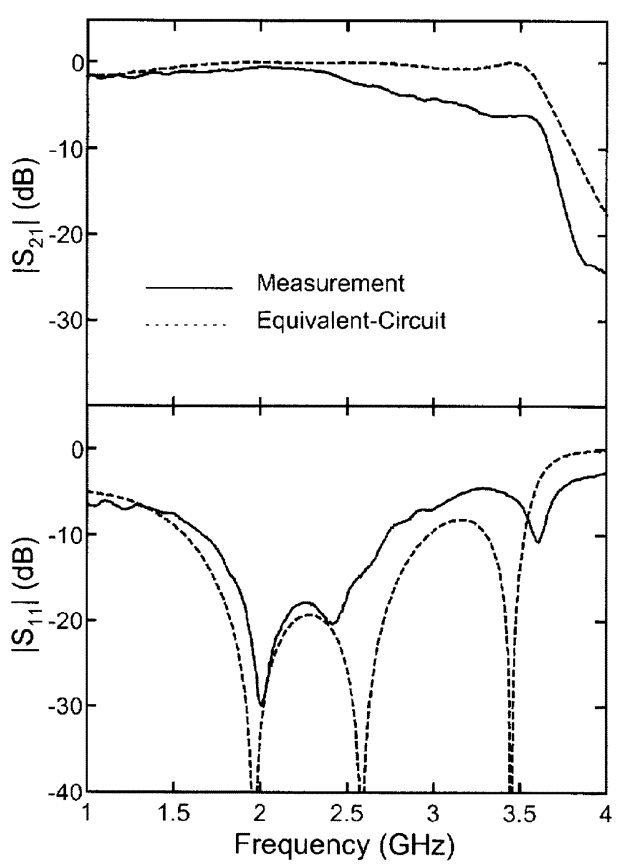

Fig. 5. Measured and simulated results of the type-I transition [see Fig. 2(a)] in back-to-back configuration with a $24-\mathrm{mm}$ CPS line in between.

line length between the two transitions in the back-to-back configuration is $24 \mathrm{~mm}$, measured from the outer edges of $\mathrm{CPW}$ ground planes. The required $L$ and $C$ values are first obtained through the Smith chart calculations and are then fine tuned to give $L_{s}=3.7 \mathrm{nH}$ and $C_{p}=0.52 \mathrm{pF}$. The corresponding geometrical parameters are obtained through the closed-form expressions. The shorter metal strip has a length of $4.8 \mathrm{~mm}$ and a width of $0.3 \mathrm{~mm}$. The five-finger interdigital capacitor has a finger width of $0.5 \mathrm{~mm}$, finger length of $1.7 \mathrm{~mm}$, and gap width of $0.2 \mathrm{~mm}$.

The back-to-back transition is measured on the Agilent 8722 network analyzer with thru-reflect-line (TRL) calibration to the CPW-CPS junction. The measured and simulated results are shown in Fig. 5. The measured insertion loss is less than $1 \mathrm{~dB}$ from 1.74 to $2.34 \mathrm{GHz}$. The return loss is greater than $20 \mathrm{~dB}$ in the 1.93-2.14-GHz frequency range. The match between measured and simulated results is good around $2 \mathrm{GHz}$. The discrepancy between measured and simulated insertion losses at higher frequencies is due to that the losses are not taken into account in the equivalent-circuit model. In addition, the back-to-back connection introduces the unwanted parasitic resonance within the CPS line between the two transitions around $3.5 \mathrm{GHz}$, which causes additional losses that are not observed in a single transition. Although the transition bandwidth is not wide due to the nature of $L$-section matching network, the total area occupied by $L_{s}$ and $C_{p}$ is only approximately $\left(\lambda_{g} / 20\right) \cdot\left(\lambda_{g} / 20\right)$, which is much smaller than those of the conventional impedance-transforming transitions based on $\lambda / 4$ line sections.

Another type-I transition for Fig. 3(a) is also built on an FR4 substrate to transform the $50-\Omega \mathrm{CPW}$ to $100-\Omega \mathrm{CPS}$. The line dimensions of the CPW and CPS are the same as those in Fig. 5. The calculated values are $C_{s}=1.18 \mathrm{pF}$, and $L_{p}=6.8 \mathrm{nH}$. Their corresponding geometrical parameters 


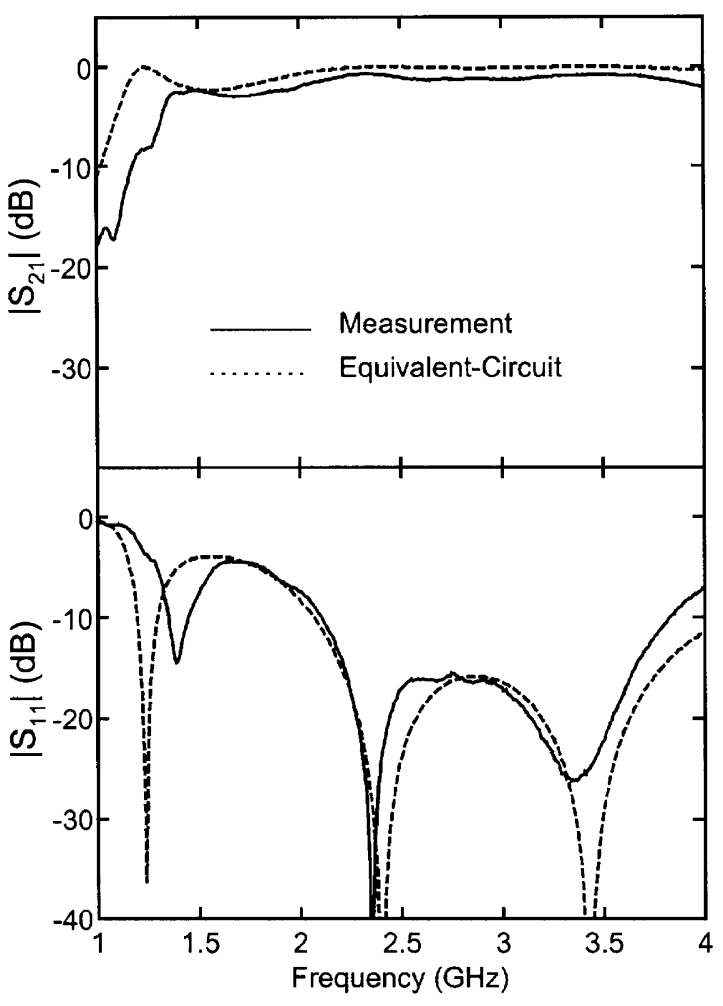

Fig. 6. Measured and simulated results of the type-I transition [see Fig. 3(a)] in a back-to-back configuration with a $24-\mathrm{mm}$ CPS line in between.

are obtained through the closed-form expressions. The shorter metal strip has a total length of $7.4 \mathrm{~mm}$ and a width of $0.2 \mathrm{~mm}$. The seven-finger interdigital capacitor has a finger width of $0.5 \mathrm{~mm}$, a finger length of $4.3 \mathrm{~mm}$, and a gap width of $0.2 \mathrm{~mm}$. The measured and simulated results are shown in Fig. 6. The measured insertion loss is less than $1 \mathrm{~dB}$ from 2.2 to $2.5 \mathrm{GHz}$. The return loss is greater than $20 \mathrm{~dB}$ in the 2.28-2.44-GHz frequency range. The match between measured and simulated results is good around the center frequency despite the discrepancy in insertion loss. The larger discrepancy between measured and simulated results around $1.3 \mathrm{GHz}$ is attributed to the power losses associated with the unwanted parasitic resonance in the back-to-back configuration that are not included in the equivalent-circuit model. This resonance occurs at a lower frequency compared to the transition for Fig. 5 because the CPS line between two transitions (Fig. 3) in the back-to-back configuration is now loaded by inductive reactances at its two ends. Anyway, the equivalent-circuit model has the advantage of very short calculation time since all the elements in the equivalent-circuit model are characterized by closed-form expressions. The total area occupied by $C_{s}$ and $L_{p}$ is only approximately $\left(\lambda_{g} / 20\right) \cdot\left(\lambda_{g} / 18\right)$ for this transition structure.

As mentioned above, the proposed impedance-transforming transition is not limited to the transformation of real impedance values, as in the case of the $\lambda / 4$ transformer. Within the realizable range of the lumped elements, the proposed impedancetransforming transitions [see Figs. 2(a) and 3(a)] may transform the complex load impedance $Z_{L}$ to $Z_{\mathrm{cpw}}$ if the normalized load impedance is inside the $1+j x$ circle on the Smith chart.

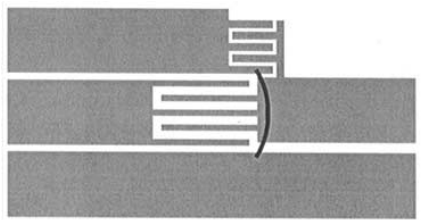

(a)

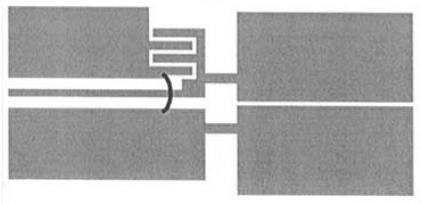

(c)

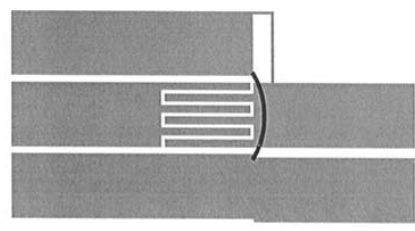

(b)

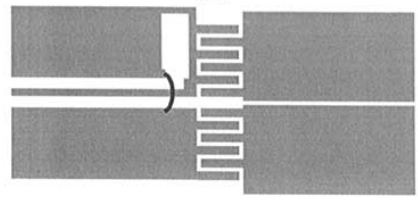

(d)
Fig. 7. Type-II impedance-transforming CPW-to-CPS transitions to realize the $L$-section networks in Fig. 1(a)-(d), respectively.

\section{B. Type-II Structures}

Shown in Fig. 7(a)-(d) are the alternative designs for the impedance-transforming CPW-to-CPS transitions. They are to realize the $L$-section networks in Fig. 1(a)-(d), respectively. Here, the asymmetric CPW-CPS junction, instead of the CPW-CPS cross-junction, is adopted as the kernel of the transition, and the interdigital capacitors and metal strip inductors are used to realize the $L$-section impedance-transforming network in the transition structures. The type-II transitions have the same orientation for CPW and CPS compared to the type-I structures, thus, may facilitate the circuit layout.

Specifically, for the transition in Fig. 7(a) or (b), the CPW is first connected to the series inductor or capacitor formed on the center conductor of the CPW, respectively. The shunt capacitor or inductor is then connected to one slot of the CPW, while the other slot of the CPW is connected to the CPS. Here, the series inductor in Fig. 7(a) is realized by the folded SL structure formed on the center conductor of CPW, which is adopted from [20]. For the transition shown in Fig. 7(c) or (d), the CPW is first connected to the shunt interdigital capacitor or metal strip inductor, respectively. It is then connected to the CPS through the series metal strip inductor or interdigital capacitor. Their corresponding equivalent-circuit models can be constructed in a similar way as type-I transitions, with the CPW-CPS cross-junction model being replaced by the asymmetric-junction model [21]. Within the realizable range of lumped elements, these transitions may provide impedance transformation to any complex load impedance within the Smith chart.

\section{Antenna Applications}

The proposed impedance-transforming CPW-to-CPS transitions can be implemented in the design of compact feeding structures for balanced antennas. Fig. 8 shows the layout of a CPW-fed bow-tie antenna using the proposed CPW-to-CPS transition as the feeding structure. The antenna structure is adopted from [22], which is composed of a pair of equilateral triangular patches and a $\mathrm{V}$-shaped dipole director to achieve an end-fire radiation pattern. In [22], a third-order microstrip-to-CPS Marchand balun was used to match the $50-\Omega$ microstrip line to the antenna input impedance. From the simulated results by HFSS, shown in Fig. 9, the antenna input impedance is $117-j 11.8 \Omega$ at $2 \mathrm{GHz}$. Here, in Fig. 8, 


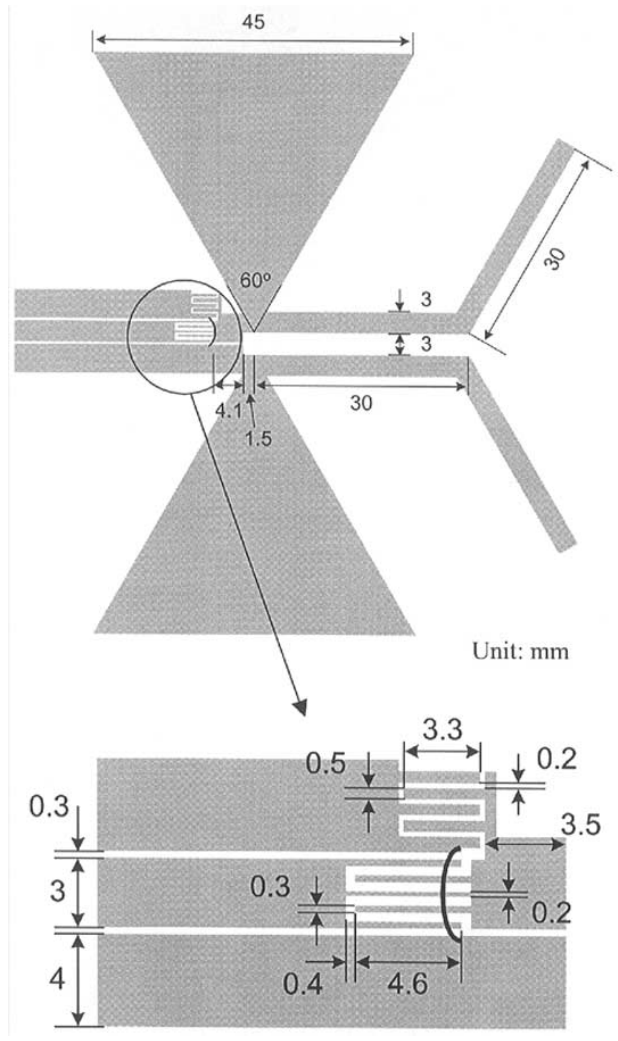

Fig. 8. CPW-fed bow-tie antenna using the proposed impedance-transforming CPW-to-CPS transition [see Fig. 7(a)] as the feeding structure.

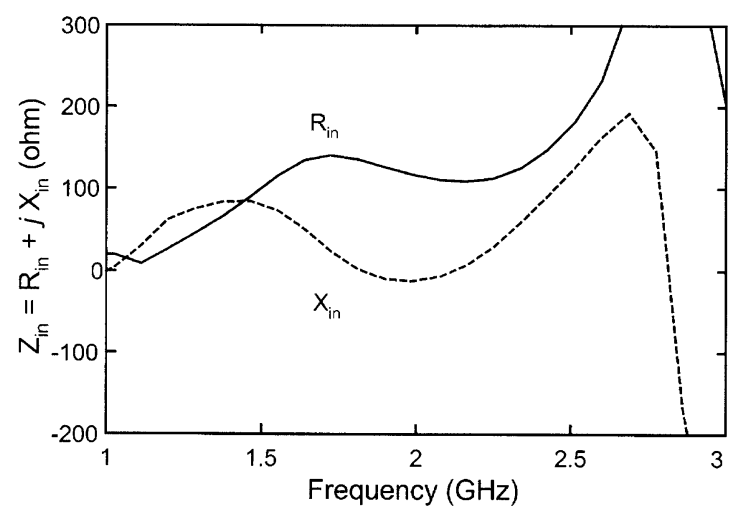

Fig. 9. Simulated input impedance of the bow-tie antenna by HFSS.

we use the impedance-transforming CPW-to-CPS transition in Fig. 7(a) instead to transform the $50-\Omega \mathrm{CPW}$ to this antenna input impedance. Design of this impedance-transforming transition can then be simply done in three steps. The initial value of $L_{s}$ and $C_{p}$ are determined as $L_{s}=4.6 \mathrm{nH}$ and $C_{p}=0.72 \mathrm{pF}$ through the Smith-chart calculations. The antenna and transition with $C_{p}$ only is then simulated using the full-wave simulator, e.g., HFSS, and the capacitance is fine tuned until the input impedance around the resonant frequency coincides with the $r=1$ circle on the Smith chart, as shown in Fig. 10. After $C_{p}$ is fixed, the inductance $L_{s}$ is added to the full-wave simulation, and is fine tuned until a good matching is obtained. The design of impedance-transforming transition for the antenna is then completed.

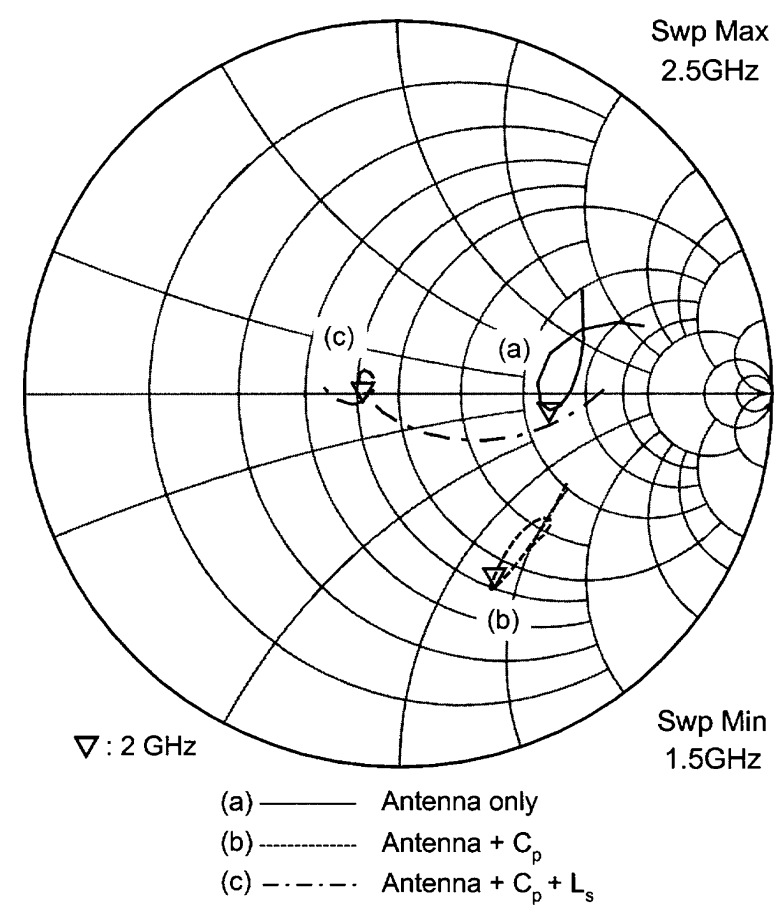

Fig. 10. Design steps for matching the CPW-fed bow-tie antenna shown in Fig. 8. The marker points are at $2 \mathrm{GHz}$.

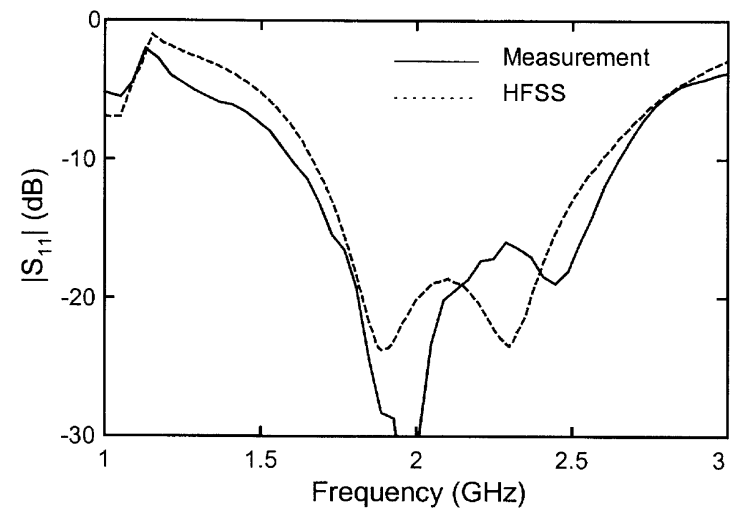

Fig. 11. Measured and simulated results for the CPW-fed bow-tie antenna (Fig. 8).

A CPW-fed bow-tie antenna for Fig. 8 is fabricated on an FR4 substrate, with the designed center frequency at $2 \mathrm{GHz}$. The measured return loss and the simulated one by HFSS are shown in Fig. 11. Good agreement between them is observed. The antenna exhibits a minimum return loss of $-36.2 \mathrm{~dB}$ at $1.97 \mathrm{GHz}$. The frequency band for $-10 \mathrm{~dB}$ return loss is from 1.6 to $2.65 \mathrm{GHz}$, corresponding to a $49.4 \%$ bandwidth. The measured radiation patterns are shown in Fig. 12, and are end-fire in shape, as expected. The measured peak gain is $4.4 \mathrm{dBi}$. In comparison to the original design [22] that utilized a third-order microstrip-to-CPS Marchand balun for impedance matching, the CPW-fed one (Fig. 8) that adopts the proposed impedancetransforming transition demonstrates a larger impedance bandwidth $(47.7 \%$ for [22]) with only a one-tenth smaller matching circuit area.

Fig. 13 shows another example of using the proposed impedance-transforming transition in the design of a CPW-fed 


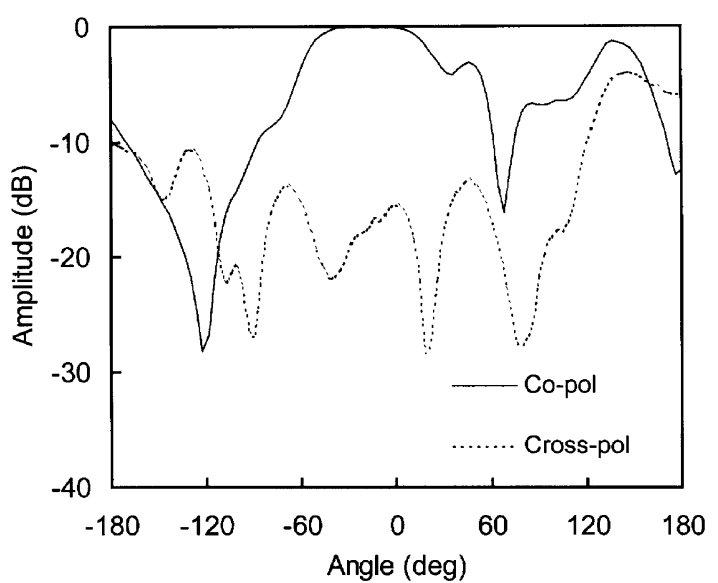

(a)

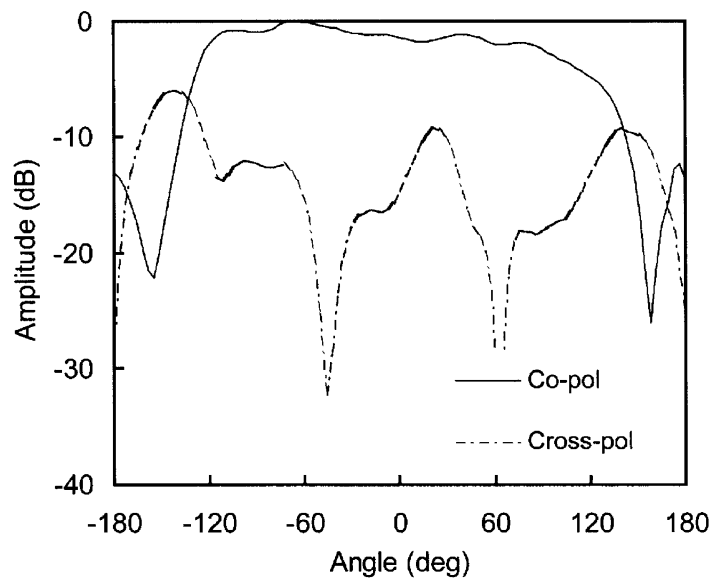

(b)

Fig. 12. Measured radiation patterns for the CPW-fed bow-tie antenna (Fig. 8) at $2 \mathrm{GHz}$. (a) $E$-plane. (b) $H$-plane.

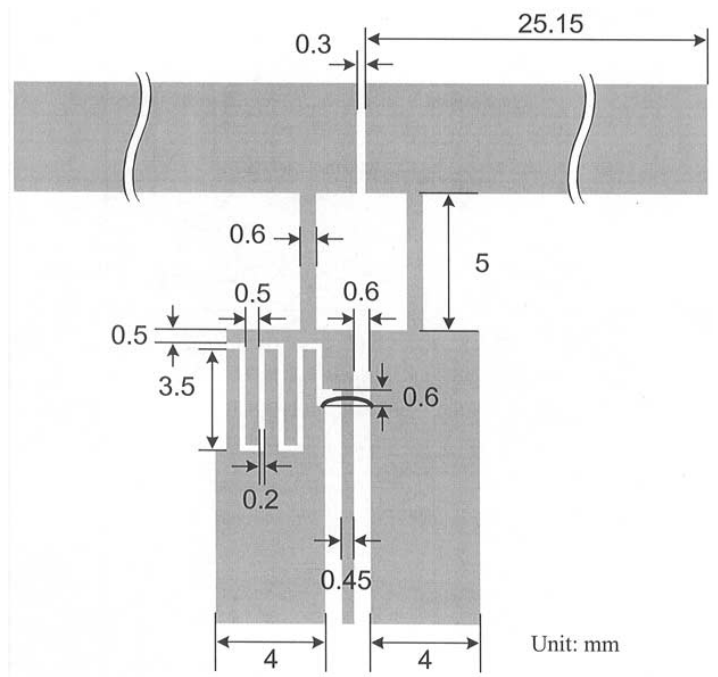

Fig. 13. CPW-fed printed dipole antenna using the proposed impedance-transforming CPW-to-CPS transition [see Fig. 7(c)] as the feeding structure.

printed dipole antenna. The simulated dipole input admittance is shown in Fig. 14(a). The first resonance frequency of the dipole is at $2.1 \mathrm{GHz}$, with an input admittance of $0.01345+j 0.007361 / \Omega$, which corresponds to an input

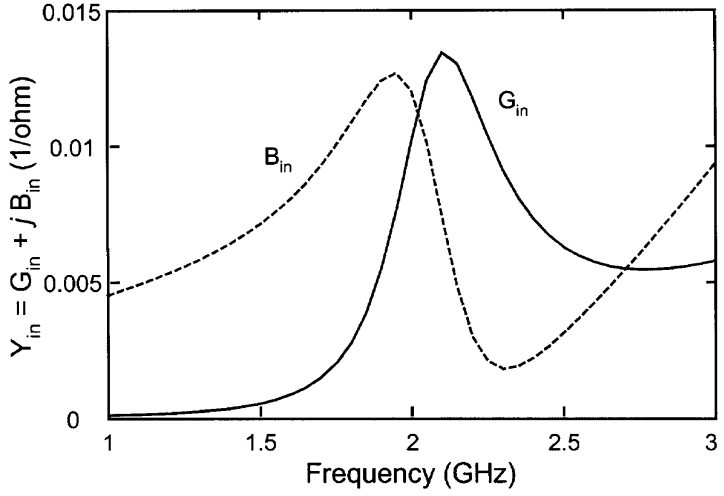

(a)

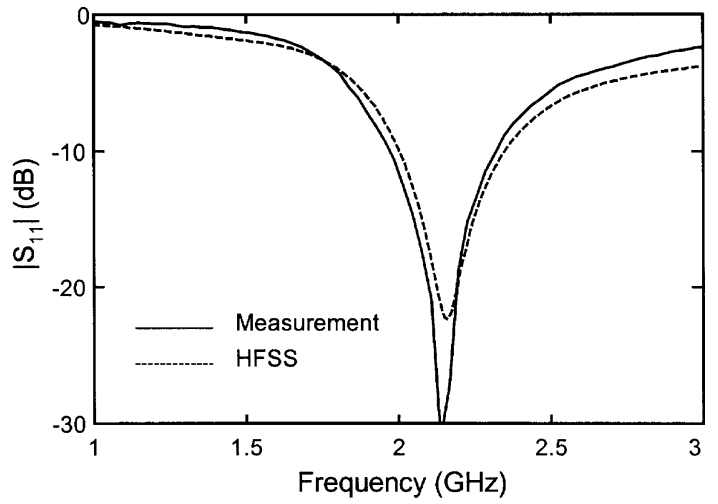

(b)

Fig. 14. (a) Simulated input admittance of the printed dipole antenna by HFSS. (b) Measured and simulated results for the CPW-fed printed dipole antenna (Fig. 13).

impedance of $57.15-j 31.32 \Omega$. The characteristic impedance of the CPW is designed to be $100 \Omega$, therefore, the transition in Fig. 7(c) is adopted to transform the $100-\Omega$ CPW to the lower dipole input impedance. A CPW-fed printed dipole for Fig. 13 is fabricated on an FR4 substrate, with the corresponding geometrical parameters shown in the same figure. The center frequency is designed to be $2.1 \mathrm{GHz}$. The TRL calibration technique is adopted in measurement to change the reference impedance to the $\mathrm{CPW}$ characteristic impedance of $100 \Omega$. The measured return loss and the simulated one by HFSS are shown in Fig. 14(b). Good agreement between them is observed. The antenna exhibits a minimum return loss of $-31.2 \mathrm{~dB}$ at $2.14 \mathrm{GHz}$. The frequency band for $-10-\mathrm{dB}$ return loss is from 1.97 to $2.32 \mathrm{GHz}$, corresponding to a bandwidth of $16.3 \%$. The total length of the impedance-transforming transition for Fig. 13 is only $\lambda_{g} / 12$ long, making the proposed CPW-fed dipole antenna one-third smaller than the conventional ones utilizing $\lambda / 4$ lines for impedance matching. In addition, the proposed transition can be easily adjusted to match the complex input impedance of antenna, such that a better impedance matching can be achieved.

By adopting the proposed impedance-transforming CPW-to-CPS transitions in the antenna feeding structures, one may largely reduce the circuit area compared to those of the conventional feeding structures based on $\lambda / 4$ lines. Although the proposed impedance-transforming transitions have narrow bandwidths, the above examples demonstrate that their bandwidths are sufficient for most antenna applications. 


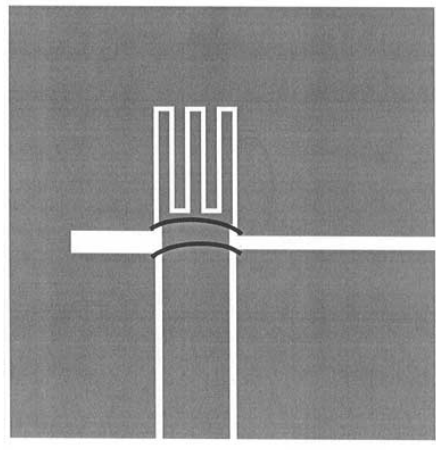

(a)

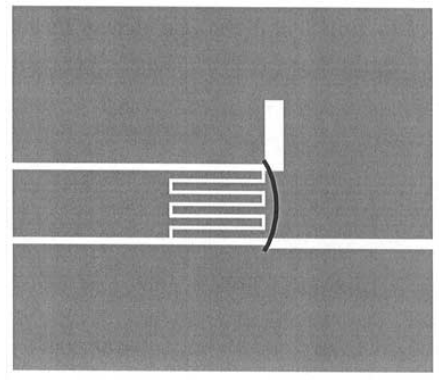

(b)

Fig. 15. Impedance-transforming CPW-to-SL transitions to realize the $L$-section network in Fig. 1(b). (a) Type I. (b) Type II.

\section{IMPEDANCE-TRANSFORMING CPW-TO-SL TRANSITIONS}

\section{A. Transition Structures}

Based on the same concept, the impedance-transforming CPW-to-SL transitions may also be built. Shown in Fig. 15 are the proposed type-I and type-II impedance-transforming CPW-to-SL transitions modified from their CPW-to-CPS counterparts [see Figs. 3(a) and 7(b)]. Let the load impedance connected to the SL port be $Z_{L}$, the proposed transitions [see Fig. 15(a) and (b)] are capable of transforming $Z_{L}$ to $Z_{\text {cpw }}$ for $z_{L}=Z_{L} / Z_{\mathrm{cpw}}$ inside the $1+j x$ circle on the Smith chart. Their corresponding equivalent-circuit models can be constructed in a similar way as their CPW-to-CPS counterparts.

Since it is not possible to realize a shunt capacitor in the SL case due to the infinitely extended ground plane, the transitions with a first series inductor as shown in Figs. 2(a) and 7(a) are not realizable here for the CPW-to-SL transition. In addition, the transitions with a first shunt reactive element as shown in Fig. 7(c) and (d) are not realizable in the SL case either.

\section{B. Antenna Applications}

Although the two impedance-transforming CPW-to-SL transitions [see Fig. 15(a) and (b)] are limited to the transformation of $z_{L}$ inside the $1+j x$ circle, they are sufficient for most applications since the SL usually has a higher characteristic impedance. Here is an example using the proposed impedance-transforming CPW-to-SL transition [see Fig. 15(b)] in the design of a CPW-fed linearly tapered slot antenna (LTSA). Shown in Fig. 16 is the layout of this CPW-fed LTSA, together with its corresponding geometrical parameters. The antenna is built on an FR4 substrate, with the designed center frequency at $5.8 \mathrm{GHz}$. The impedance-transforming CPW-to-SL transition is designed to transform the $50-\Omega \mathrm{CPW}$

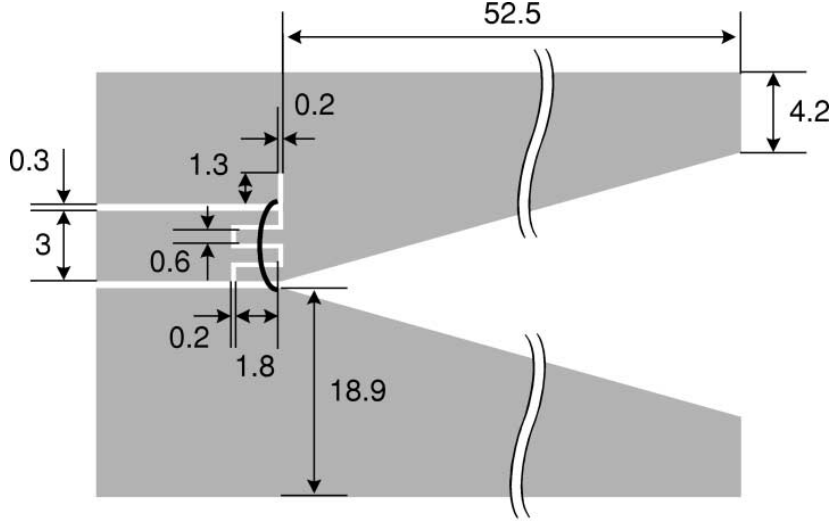

Unit: mm

Fig. 16. CPW-fed LTSA using the proposed impedance-transforming CPW-to-SL transition [see Fig. 15(b)] as the feeding structure.

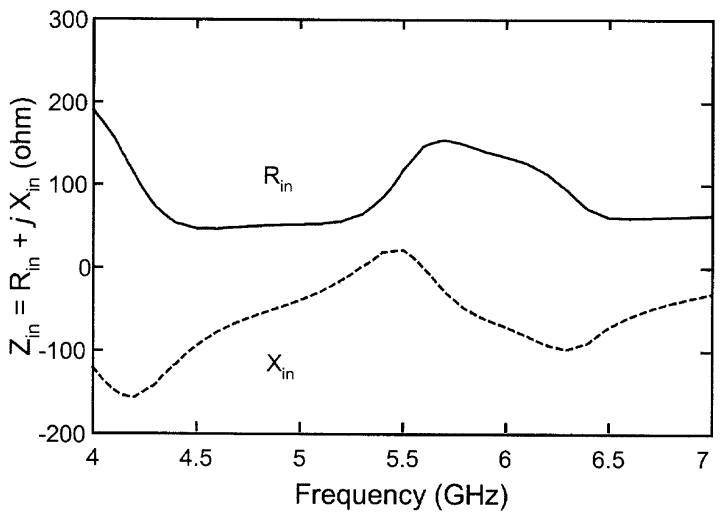

(a)

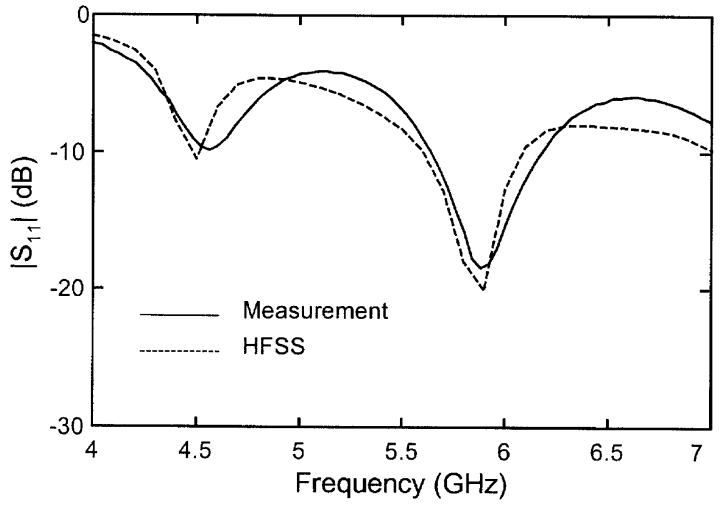

(b)

Fig. 17. (a) Simulated input impedance of the LTSA by HFSS. (b) Measured and simulated results for the CPW-fed LTSA (Fig. 16).

to the antenna input impedance whose simulated value is $148-j 49.1 \Omega$ at $5.8 \mathrm{GHz}$ [see Fig. 17(a)]. For the CPW-fed LTSA structure, the measured and simulated results are shown in Fig. 17(b). The measured return loss is less than $-10 \mathrm{~dB}$ from 5.64 to $6.18 \mathrm{GHz}$, which is compliant to the industrial-scientific-medical (ISM) band regulations $(5.725-5.85 \mathrm{GHz})$. The measured radiation patterns are shown in Fig. 18, with a peak gain of $7.1 \mathrm{dBi}$. The total length of the impedance-transforming transition for this CPW-fed LTSA (Fig. 16) is only $\lambda_{g} / 17$ long.

Although the bandwidth is narrower compared to other reported LTSA with different feeding networks [23], [24], the 


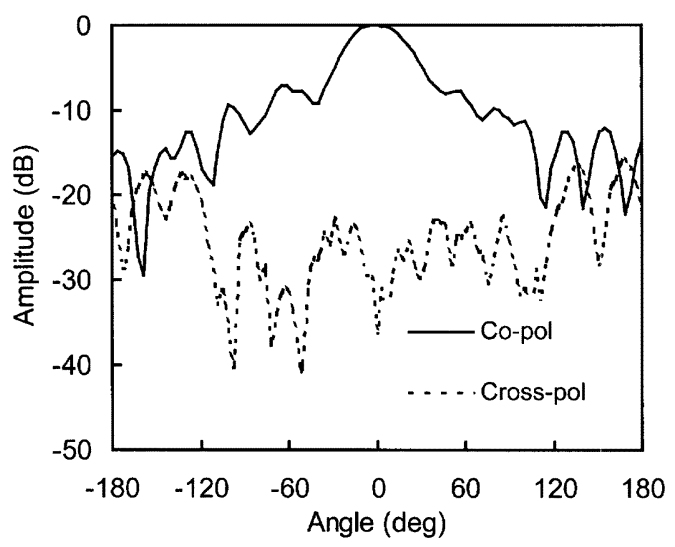

(a)

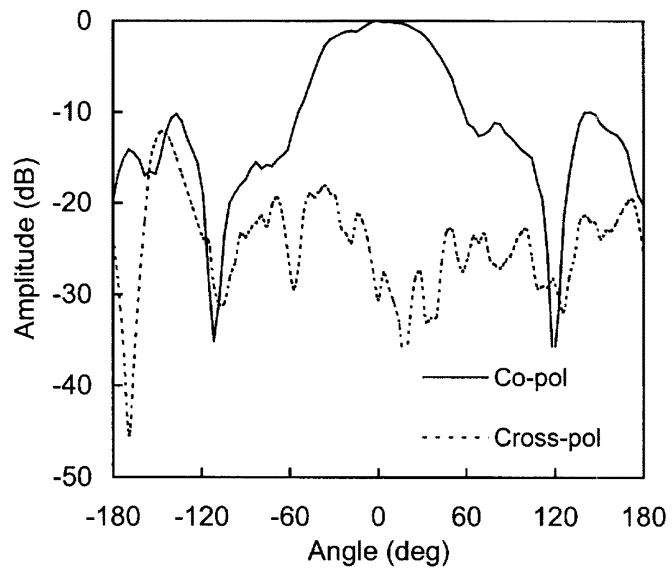

(b)

Fig. 18. Measured radiation patterns for the CPW-fed LTSA (Fig. 16) at 5.8 GHz. (a) $E$-plane. (b) $H$-plane.

proposed CPW-fed LTSA utilizing the impedance-transforming CPW-to-SL transition has the advantages of very compact size such that it can facilitate circuit layout especially in LTSA array applications. In addition, it has the merit of versatile design capability for complex impedance matching. These merits make the proposed transitions attractive for narrow-band applications.

All the transitions proposed in Sections II and III are based on the $L$-section matching networks, therefore, their bandwidths depend on the impedance ratio between the source and load, and are in the order of 10\%-50\%. Compared to other conventional impedance-transforming transition/balun designs, the proposed transition has a bandwidth comparable to those of the $\lambda / 4$ transformer-type transitions/baluns. The bandwidth of proposed transition is less than those of the impedance transforming Marchand baluns in [3], [14], and [15], which use the third- or fourth-order structures and may achieve larger than 2: 1 bandwidth in general. Regardless, the sizes of proposed transitions are much smaller and may provide impedance transformation between complex impedance values.

\section{CONCLUSIONS}

In this paper, novel lumped-element impedance-transforming CPW-to-CPS and CPW-to-SL transitions have been proposed, using planar lumped elements to realize the L-section impedance-matching network in the transition structure. The proposed transitions are very compact and not limited to the transformation of real impedance values, as the conventional impedance-transforming transitions based on $\lambda / 4$ lines. For design purpose, simple equivalent-circuit models based on closed-form expressions have also been established. The proposed transitions may then be easily designed through the basic knowledge on impedance transformation and equivalent-circuit models. Applications of the proposed transitions to the antenna feeding structures have also been demonstrated. The resulting antenna feeding structures are very compact, thus, they may reduce the complexity of the feeding network in antenna array applications. The proposed transitions provide a simple way to accomplish impedance transformation for narrow bandwidth, and may find applications in uniplanar MMICs such as antenna feeding structures and balanced mixers.

\section{REFERENCES}

[1] K. C. Gupta, R. Garg, and I. J. Bahl, Microstirp Lines and Slotlines, 2nd ed. Norwood, MA: Artech House, 1996, ch. 5, 7

[2] C.-H. Ho, L. Fan, and K. Chang, "Experimental investigations of CPWslotline transitions for uniplanar microwave integrated circuits," in IEEE MTT-S Int. Microwave Symp. Dig., 1993, pp. 877-880.

[3] V. Trifunović and B. Jokanovic', "Review of printed Marchand and double Y baluns: Characteristics and application," IEEE Trans. Microwave Theory Tech., vol. 42, pp. 1454-1462, Aug. 1994.

[4] K. Hettak, N. Dib, A. Sheta, A. A. Omar, G. -Y. Delisle, M. Stubbs, and S. Toutain, "New miniature broad-band CPW-to-slotline transitions," IEEE Trans. Microwave Theory Tech., vol. 48, pp. 138-146, Jan. 2000.

[5] S.-G. Mao, C.-T. Hwang, R.-B. Wu, and C. H. Chen, "Analysis of coplanar waveguide-to-coplanar stripline transitions," IEEE Trans. Microwave Theory Tech., vol. 48, pp. 23-29, Jan. 2000.

[6] L. Zhu and K. Wu, "Hybrid FGCPW/CPS scheme in the building block design of low-cost uniplanar and multilayer circuit and antenna," in IEEE MTT-S Int. Microwave Symp. Dig., 1999, pp. 867-870.

[7] Y.-S. Lin and C. H. Chen, "Design and modeling of twin-spiral coplanar waveguide-to-slotline transitions," IEEE Trans. Microwave Theory Tech., vol. 48, pp. 463-466, Mar. 2000.

[8] — "Novel lumped-element uniplanar transitions," IEEE Trans. Microwave Theory Tech., vol. 49, pp. 2322-2330, Dec. 2001.

[9] — "Novel lumped-element coplanar waveguide-to-coplanar stripline transitions with low-pass and high-pass characteristics," in IEEE MTT-S Int. Microwave Symp. Dig., 2002, pp. 2213-2216.

[10] _ _ "Coplanar waveguide-to-coplanar stripline transition-filter structures," in Proc. Asia-Pacific Microwave Conf., 2002, pp. 1489-1492.

[11] C.-H. Wang, Y.-S. Lin, H. Wang, and C. H. Chen, "A $Q$-band uniplanar MMIC diode mixer with lumped-element coplanar waveguide-to-slotline transition," in IEEE MTT-S Int. Microwave Symp. Dig., 2003, pp. 103-106.

[12] Y.-D. Lin and S.-N. Tsai, "Coplanar waveguide-fed uniplanar bow-tie antenna," IEEE Trans. Antennas Propagat., vol. 45, pp. 305-306, Feb. 1997.

[13] T.-H. Lin and R. B. Wu, "CPW to waveguide transition with tapered slotline probe," IEEE Microwave Wireless Comp. Lett., vol. 11, pp. 314-316, July 2001.

[14] K. S. Ang, I. D. Robertson, K. Elgaid, and I. G. Thayne, " 40 to $90 \mathrm{GHz}$ impedance-transforming CPW Marchand balun," in IEEE MTT-S Int. Microwave Symp. Dig., 2000, pp. 1141-1144.

[15] K. S. Ang and I. D. Robertson, "Analysis and design of impedancetransforming planar Marchand baluns," IEEE Trans. Microwave Theory Tech., vol. 49, pp. 402-406, Feb. 2001.

[16] D. M. Pozar, Microwave Engineering. Reading, MA: Addison-Wesley, 1993, ch. 6

[17] A. E. Ruehli, "Inductance calculations in a complex integrated circuit environment," IBM J. Res. Develop., vol. 16, pp. 470-481, Sept. 1972.

[18] S. S. Gevorgian, T. Martinsson, L. J. P. Linner, and E. L. Kollberg, "CAD models for multilayered substrate interdigital capacitors," IEEE Trans. Microwave Theory Tech., vol. 44, pp. 896-904, June 1996.

[19] M. Ribo and L. Pradell, "Circuit model for a coplanar-slotline cross," IEEE Microwave Guided Wave Lett., vol. 10, pp. 511-513, Dec. 2000. 
[20] K. Hettak, N. Dib, A.-F. Sheta, and S. Toutain, "A class of novel uniplanar series resonators and their implementation in original applications," IEEE Trans. Microwave Theory Tech., vol. 46, pp. 1270-1276, Sept. 1998.

[21] M. Ribo, J. de la Cruz, and L. Pradell, "Circuit model for slotline-tocoplanar waveguide asymmetrical transitions," Electron. Lett., vol. 35, pp. 1153-1155, July 1999.

[22] S.-G. Mao, C.-H. Chan, C.-I. G. Hsu, and D.-C. Chang, "A series-fed printed-bowtie antenna with broadband characteristics and end-fire radiation," in Proc. Asia-Pacific Microwave Conf., 2001, pp. 926-929.

[23] A. Nesic, "Endifire slotline antennas excited by a coplanar waveguide," in IEEE AP-S Int. Symp. Dig., vol. 2, 1991, pp. 700-702.

[24] R. N. Simons, R. Q. Lee, and T. D. Perl, "New techniques for exciting linearly tapered slot antennas with coplanar waveguide," Electron. Lett., vol. 28, pp. 620-621, Mar. 1992.

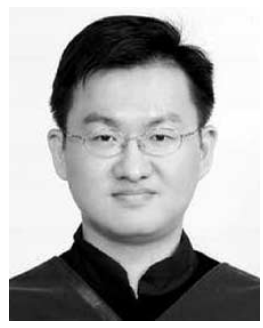

Yo-Shen Lin (M'04) was born in Taipei, Taiwan, R.O.C., in 1973. He received the B.S. and M.S.E.E. degrees in electrical engineering and Ph.D. degree in communication engineering from the National Taiwan University, Taipei, Taiwan, R.O.C., in 1996, 1998, and 2003, respectively.

From 1998 to 2001, he was with Acer Communication and Multimedia Inc., Taipei, Taiwan, R.O.C., where he designed global system for mobile communications (GSM) mobile phones. In 2001, he joined Chi-Mei Communication System Inc., Taipei, Taiwan, R.O.C., where he was involved with the design of low-temperature co-fired ceramic (LTCC) RF transceiver module for GSM mobile applications. $\mathrm{He}$ is currently a Post-Doctoral Research Fellow with the Graduate Institute of Communication Engineering, National Taiwan University. His research interests include the design and analysis of planar microwave circuits and RF transceiver module for wireless communication systems.

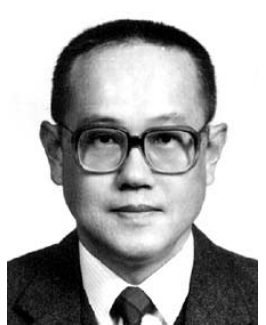

Chun Hsiung Chen (SM'88-F'96) was born in Taipei, Taiwan, R.O.C., on March 7, 1937. He received the B.S.E.E. and Ph.D. degrees in electrical engineering from the National Taiwan University, Taipei, Taiwan, R.O.C., in 1960 and 1972, respectively, and the M.S.E.E. degree from the National Chiao Tung University, Hsinchu, Taiwan, R.O.C., in 1962.

In 1963, he joined the Faculty of the Department of Electrical Engineering, National Taiwan University, where he is currently a Professor. From August 1982 to July 1985, he was Chairman of the Department of Electrical Engineering at the same university. From August 1992 to July 1996, he was the Director of the University Computer Center. In 1974, he was a Visiting Scholar with the Department of Electrical Engineering and Computer Sciences, University of California at Berkeley. From August 1986 to July 1987, he was a Visiting Professor with the Department of Electrical Engineering, University of Houston, Houston, TX. In 1989, 1990, and 1994, he visited the Microwave Department, Technical University of Munich, Munich, Germany, the Laboratoire d'Optique Electromagnetique, Faculte des Sciences et Techniques de Saint-Jerome, Universite d'Aix-Marseille III, Marseille, France, and the Department of Electrical Engineering, Michigan State University, East Lansing, respectively. His areas of interest include microwave circuit analysis and computational electromagnetics. 